Proceeding of the $1^{\text {st }}$ ICEENG conference, 24-26 March, 1998. RG6-420

MILITARY TECHNICAL COLLEGE ICEENG 98 FIRST INTERNATIONAL CONF. ON
CAIRO-EGYPT

\title{
Extraction of Frequency Domain Parameters of the Guidance System Via Its Six-Degrees-of-Freedom (6-DOF) Simulation Model
}

\begin{abstract}
'M. S. Aly and ${ }^{2}$ Y. Z. El-Halwagy
ABSTRACT

Mathematical modeling constitutes one of the recognized simulation techniques that can be employed in systems analysis. In an earlier work [1], a six degrees-of-freedom simulation model had been utilized for missile airframe resonances extraction from its transient response. In the present work, the complete transfer function of the airframe is predicted. The analysis is extended to cover the missile autopilot. The obtained results show that the missile airframe can accurately be described by a linear second order system with two complex conjugate poles and two complex conjugate zeros. The airframe characteristics are sensitive to speed variations. However, this effect can be reduced by the utilization of autopilot with accelerometer and rate gyro in the feed back path.
\end{abstract}

\section{INTRODUCTION}

Mathematical model, in its most general definition, is a mathematical description of a physical system or process. In its simplest and common form, it is a set of differential or difference equations whose inputs correspond to the physical stimuli given to the system and whose outputs describe the behavior of the system. In particular, it determines whether that system is successful in its design function. The importance of mathematical modeling in system design and development is increased by the continuous development of computing systems which enables the numerical solution of extremely involved models that accurately describe the physical systems

The model of present concern is for a six degrees of freedom motion of a command guided missile system (6DOF). The equations that describe the missile's center of gravity (C.G) kinematical and dynamical motion, kinematical and dynamical rotation of the missile body around its C.G, and the on-board measuring and control devices are considered. As well, the rest of the guidance loop subsystem that are located in the launching guidance site are involved. Modular structuring is followed during the development of the code that solves the model's equations. Each module describes a physically existing subsystem or process.

The missile system under consideration is guided from the launching site. The coordinates of the engaged target and the launched missile are provided through a guidance radar. The missile is aerodynamically controlled via two pairs of rear control surfaces. Communication of guidance commands to the missile is accomplished via radio command link. The missile utilizes on-board lateral autopilot with acceleration and body rate feedbacks. The issued commands are, thus, made proportional to the missile lateral acceleration. The missile motor is of boost-sustain type. The booster motor is contained in the first stage that is disposed at the end of the boost phase.

The command guidance system under consideration utilizes three-point guidance method. The method of control is based on the generation of acceleration commands proportional to the lateral displacement of the missile from the guidance station-target line-of-sight (LOS).

\footnotetext{
${ }^{1}$ Associate Professor, Department of Radar and Guidance, Military Technical College, Cairo, Egypt.

${ }^{2}$ Graduate student, Department of Radar and Guidance, Military Technical College, Cairo, Egypt.
} 
In an earlier work [1], the missile airframe had been analyzed. The weathercock undamped frequency and damping coefficient of the airframe are obtained from the airframe step response in the time domain. Also, the airframe time constant which corresponds to the delay between the airframe turn rate around its C.G. and the start of the C.G. turn in space[2]. The calculations had been made at different mach numbers given by $2,3,4$, and $5 \mathrm{M}$. It had been found that the increase in the mach number of the airframe increases its weathercock frequency and decreases its damping coefficient. The calculated airframe incidence lag time showed that it decreases by the increase of the mach number. The obtained results show that the airframe bandwidth can be fairly doubled by doubling the mach number. These findings indicate that the tactical capabilities of the missile can by enhanced significantly by increasing its speed. Thus, the utilization domain of these missile systems can be extended to cover new harder tactical scenarios.

In this paper, the analysis is extended to predict the complete transfer function of the airframe. The zero locations of the transfer function in the complex domain are calculated from the airframe step response in the time domain. The autopilot is then analyzed. The autopilot response to unit step input acceleration command is computed via the $6 \mathrm{DOF}$ model. The frequency-domain response is calculated through the FFT of the time-domain data. The pole and zero locations of the autopilot are determined. A comparison between the airframe response and the autopilot response is made to show the advantage of having accelerometer and rate gyro.

\section{GUIDANCE SYSTEM DESCRIPTION}

System evaluation represents one of the major tasks following the system design. The importance of system evaluation is further increased if the concerned system is a missile guidance system. The evaluation nature is extended from theoretical evaluation as in the present situation to realistic evaluation through practical experimentation and field tests [3]. The developed 6DOF simulation model represents the evaluation tool. The establishment of a new control system and/or the improvement of an existing system requires complete information about the main building blocks of the system. If these building blocks are linear, the transfer function concepts have been proved to be the most successful concept [4] and [5]. For the concerned guidance system, the missile airframe and its control system are the major building blocks.

Fig. (1) shows a simplified block diagram of the missile guidance loop and the location of the autopilot loop. The deviation of the missile LOS direction from the target LOS direction is measured by the angular channel receiver. The output of the angular channel receiver is sent to the guidance computer where the guidance commands are generated according to the adopted guidance law and the instantaneous positions of the missile and target. The guidance commands, then control the missile motion in space through the missile control system or the autopilot. The difference between the demanded and actual acceleration is transmitted to a limiting device to prevent the application of high acceleration values to the missile beyond its structural capability and to assure missile frame stability throughout flight.

As shown in Fig. (2) the autopilot contains a pneumatic fin servo, one accelerometer, one rate gyro, and the conditioning electronic circuits. In the following discussion the motions of the airframe in pitch plane will only, be considered. Since the missile has a cruciform airframe, same conclusions are applied to the yaw plane. 
Proceeding of the $1^{\text {st }}$ ICEENG conference, 24-26 March, 1998. RG6-4 22

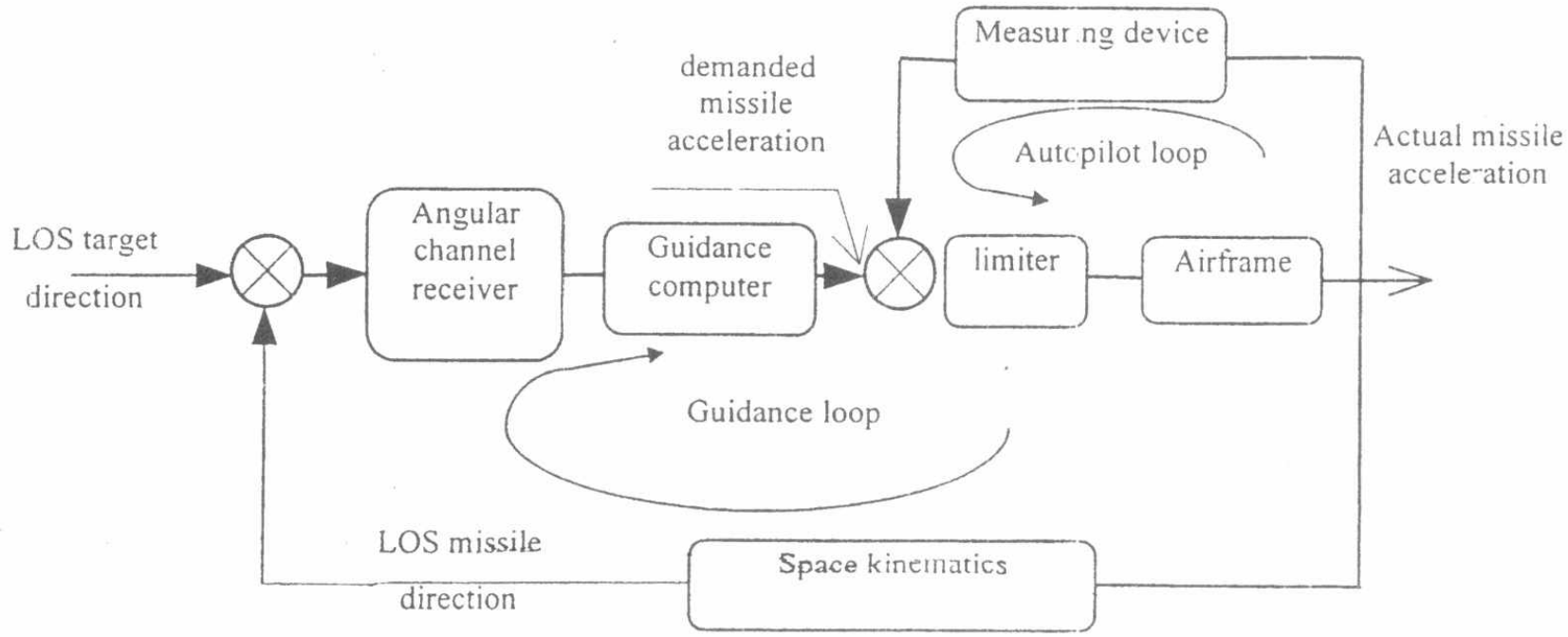

Fig. 1. Simplified block diagram of the guidance loop with autopilot block.

From the control point of view, the input to the missile airframe is the control fin deflection (aerodynamic control) and its outputs are the different flight parameters (normal acceleration, incidence angle,.....). The aerodynamic coefficients have in general nonlinear dependence on the mach number and incidence angles. However, for small incidence angles and at fixed mach number, the airframe can be fairly considered as a linear element. Hence, the transfer function concept is adequate.

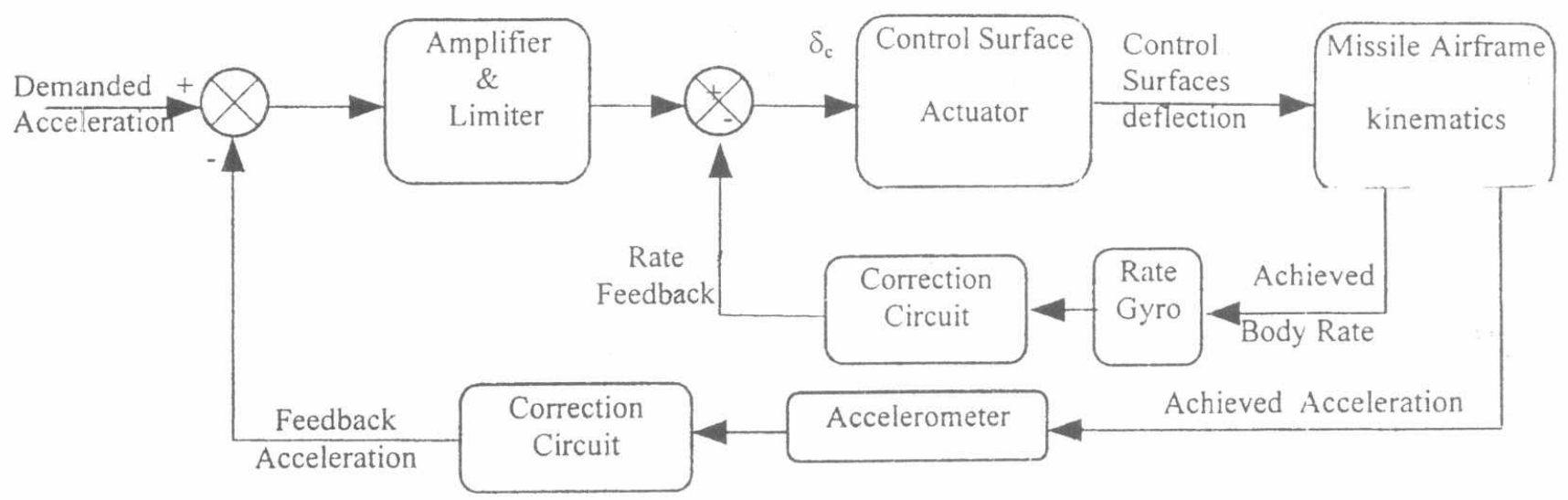

Fig. 2. Simplified block diagram of pitch autopilot

\section{MATHIEMATICAL MODEL DESCRIPTION}

The guidance law which relates the missile C.G motion to the target's motion for the three-point guidance method can be written as:

$$
\varepsilon_{\mathrm{m}}=\varepsilon_{\mathrm{t}}
$$

and

$$
\beta_{\mathrm{m}}=\beta_{\mathrm{t}},
$$

where $\varepsilon_{\mathrm{m}}, \beta_{\mathrm{m}}, \varepsilon_{\mathrm{t}}$, and $\beta_{\mathrm{t}}$ are the missile and target LOS angles. The guidance command voltages are designed to be proportional to the linear deviations of the missile C.G from the target-guidance point LOS. Thus, the commanded missile acceleration is given by: 
Proceeding of the $1^{\text {st }}$ ICEENG conference, 24-26 March, 1998. RG6-42

and

$$
h_{\varepsilon}=r_{m}\left(\varepsilon_{t}-\varepsilon_{m}\right)
$$

$$
h_{\beta}=r_{m}\left(\beta_{t}-\beta_{m}\right) \cos \varepsilon_{m},
$$

where $h_{\varepsilon}$ and $h_{\beta}$ represent the linear missile deviations for the target LOS in the elevation and azimuth planes; respectively. $r_{m}$ is the missile range. The missile equations of motion can be written as [2] :

$$
\begin{aligned}
& \mathrm{T}_{\mathrm{x}}-\mathrm{F}_{\mathrm{x}}-\mathrm{mg} \sin \theta=\mathrm{m}(\dot{\mathrm{U}}+\mathrm{qw}-\mathrm{rv}) \\
& \mathrm{F}_{\mathrm{y}}+\mathrm{mg} \cos \theta \sin \varphi=\mathrm{m}(\dot{\mathrm{v}}-\mathrm{pw}+\mathrm{rU}) \\
& \mathrm{F}_{\mathrm{z}}+\mathrm{mg} \cos \theta \cos \varphi=\mathrm{m}(\dot{\mathrm{w}}-\mathrm{qU}+\mathrm{pv}) \\
& \mathrm{M}_{\mathrm{x}}=\mathrm{I}_{\mathrm{xx}} \dot{\mathrm{p}}+\mathrm{qr}\left(\mathrm{I}_{\mathrm{zz}}-\mathrm{I}_{\mathrm{yy}}\right) \\
& \mathrm{M}_{\mathrm{y}}=\mathrm{I}_{\mathrm{yy}} \dot{\mathrm{q}}+\operatorname{pr}\left(\mathrm{I}_{\mathrm{xx}}-\mathrm{I}_{\mathrm{zz}}\right) \\
& \mathrm{M}_{\mathrm{z}}=\mathrm{I}_{\mathrm{zz}} \dot{\mathrm{r}}+\mathrm{pq}\left(\mathrm{I}_{\mathrm{yy}}-\mathrm{I}_{\mathrm{xx}}\right)
\end{aligned}
$$

Where U, v, and $w$ are the velocity components along the missile's axes as shown in Fig.(3). p, q, and $r$ are the missile angular rates. $F_{X}, F_{y}, F_{z}, M_{X}, M_{y}$, and $M_{z}$ are the aerodynamic forces and moments that are given by :

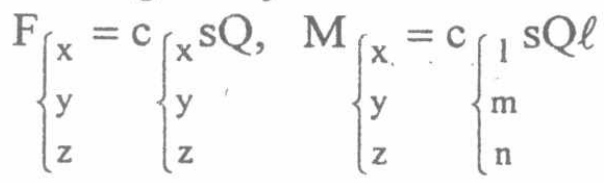

Where $c_{x}, c_{y}, c_{z}$, and $c_{l}, c_{m}, c_{n}$, respectively are the aerodynamic force and moment coefficients that describe the missile airframe and are given by

and

$$
\begin{aligned}
& c_{x}=c_{x o}+c_{x}^{\alpha} \alpha^{2}, \\
& c_{y}=c_{y}^{\beta} \beta+c_{y}^{\delta} \delta_{r}, \\
& c_{z}=c_{z}^{\alpha} \alpha+c_{z}^{\delta} \delta_{e}, \\
& c_{l}=c_{l p} p, \\
& c_{m}=c_{m \alpha} \alpha+c_{m \delta} \delta+c_{m q} q,
\end{aligned}
$$

$$
c_{n}=c_{n \beta} \beta+c_{n \delta} \delta+c_{m r} r .
$$

Where $\mathrm{s}$ is the reference cross-sectional area and is given by $\pi \mathrm{d}^{2} / 4$, with $\mathrm{d}$ being the missile reference diameter. Fig. (3). shows the orientation of these variables in the airframe coordinate system. $Q$ is the atmospheric dynamic pressure that is given by $\frac{1}{2} \rho v_{m}^{2}$, where $\rho$ is the air density and $\mathrm{V}_{\mathrm{m}}$ is the total missile velocity. $\ell$ is the missile reference length. The aerodynamic forces and moments coefficients are calculated and stored aperiori as functions of the missile velocity [6]. 


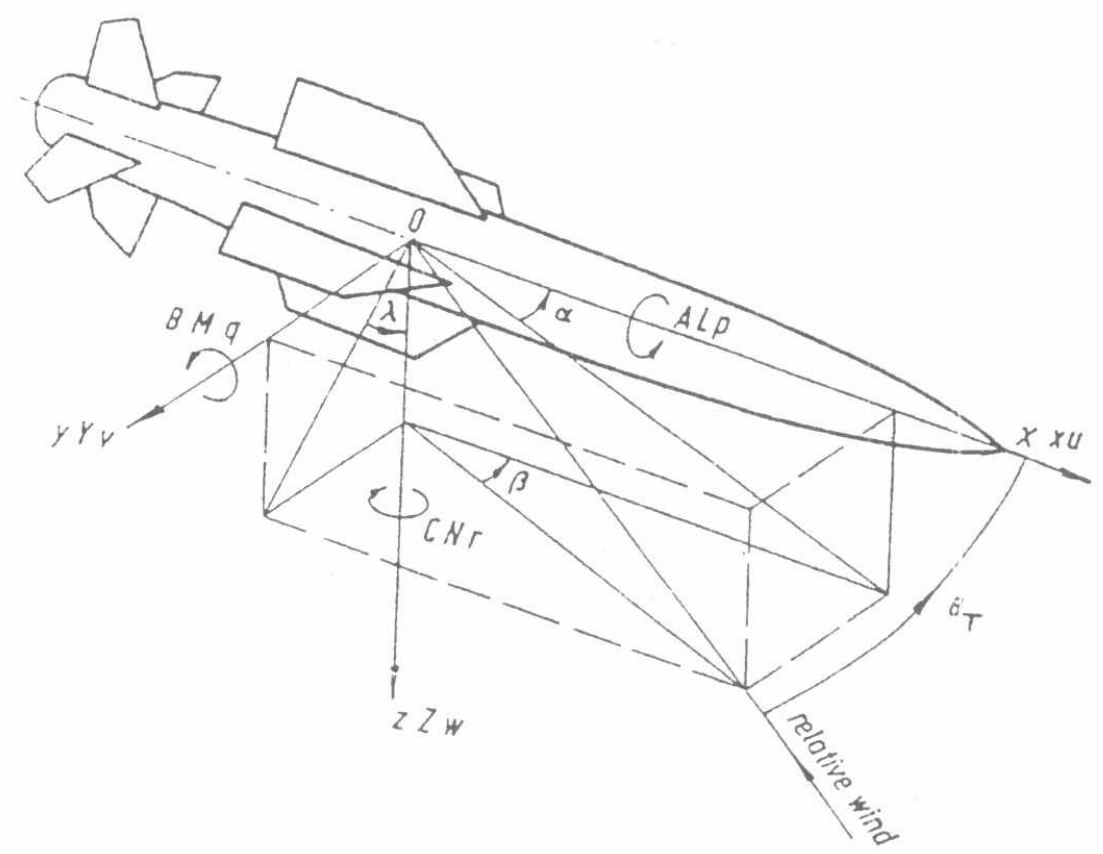

Fig. (3) Force, Moment, etc., Conventions.

\section{6-DOF SIMULATION MODEL DESCRIPTION}

A simplified block diagram of the simulation model is shown in Fig. (4). The simulation model is broken down into the following major parts: missile-target geometry, guidance, autopilot, airframe, and kinematics.

In the missile-target geometry module, the missile position relative to the target is calculated. The guidance parameters which are the deviation errors between the ideal and actual position of the missile measured by guidance radar are then calculated. The guidance module receives the guidance parameters and generates the guidance steering command signals. The guidance module performs all the necessary signal processing required for shaping the missile steering commands. The signal processing includes compensations, limitations and various signals summations. The guidance signals are supplied to the autopilot to steer the missile in space.

In the airframe module various forces and moments are calculated. They involve aerodynamic, weight, thrust, and control forces and moments. The aerodynamics forces and moments are calculated in the velocity coordinate system. However the thrust and weight forces are computed in the board and reference coordinate systems; respectively. Thus, the solution of the dynamical problem necessitates a reliable means for coordinates transformations between these system. The transformations between these coordinate systems achieved by the Euler's angles method. Finally, the kinematics module solves the force and moment equations and produces the missile flight parameters which are the instantaneous acceleration, velocity, and posit on data of the missile. The flight path variables are, then derived from the airframe module. Fig. (5) shows the data flow among the various routines that constitutes the simulation code [7][8]. 
Proceeding of the $1^{\text {st }}$ ICEENG conference, 24-26 March, 1998. RG6-425

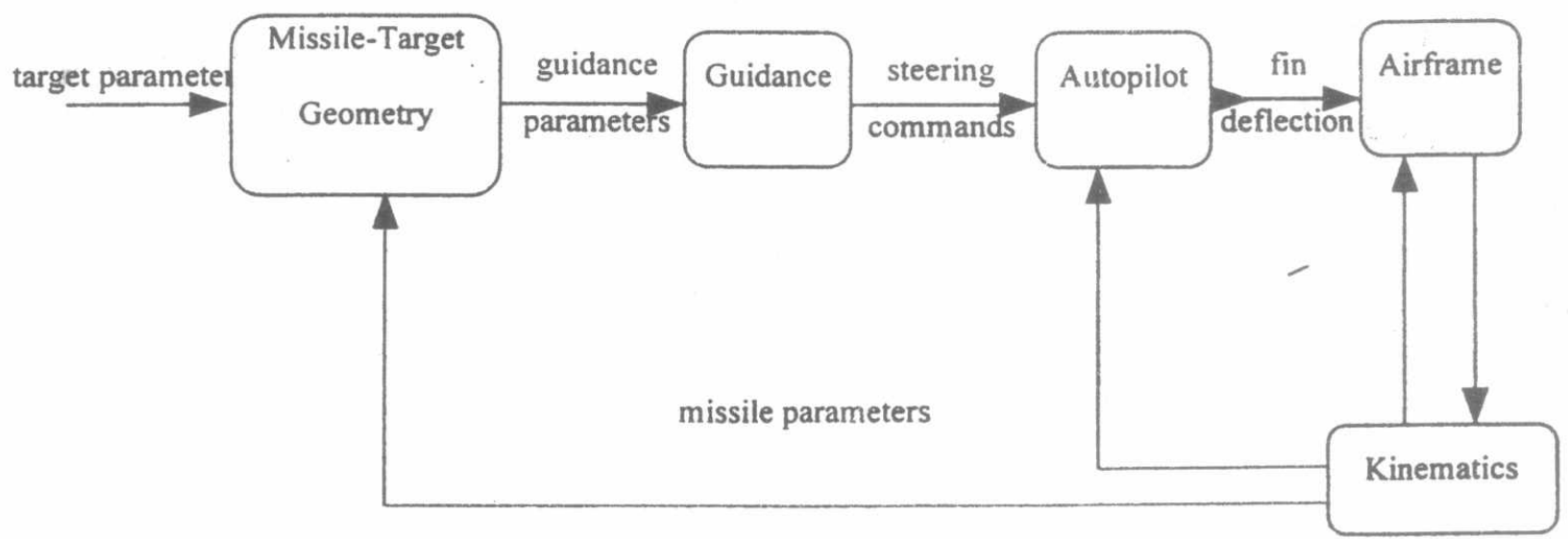

Fig. (4) Simplified block diagram of the main simulation model modules.

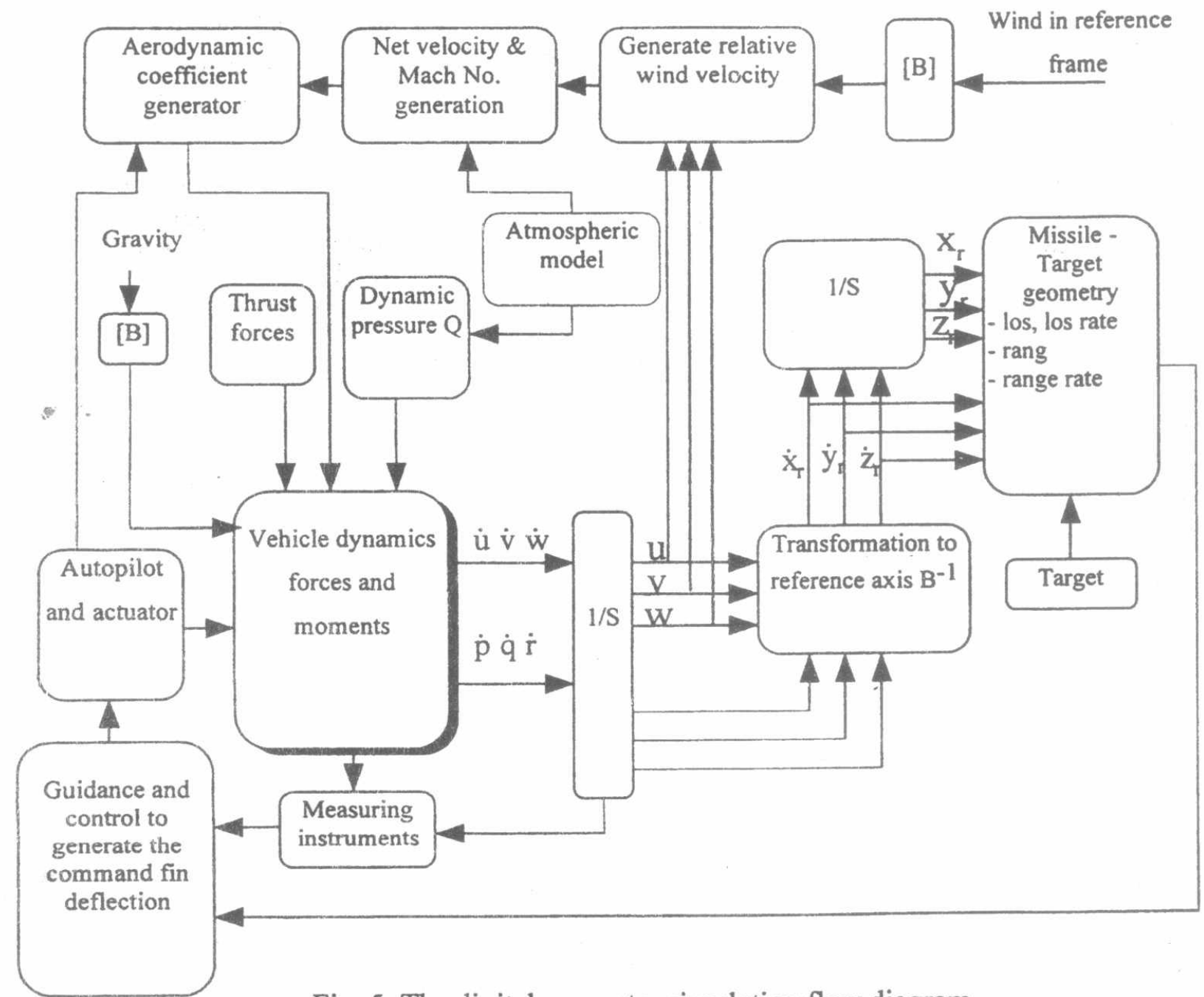

Fig. 5. The digital computer simulation flow diagram. 
Proceeding of the $1^{\text {st }}$ ICEENG conference, 24-26 March, 1998. RG6-4 26

\section{RESLLTS AND ANALYSIS}

A computer code written in BORLAND C is constructed to solve the simulation model. The input stimuli to the code are the launch conditions and target trajectory considerations. The outputs are the missile flight data (speed, acceleration, range and body turn rates). In the previous work [1 ]. the unit step response of the missile airframe had been calculated at different mach numbers. The response had been approximated by a second order lag system response with two poles only viz:

$$
\begin{aligned}
& \frac{f_{z}}{\delta}=\frac{k_{b 1}}{s^{2} / \omega_{n b}^{2}+2 \xi / \omega_{n b}+1} . \\
& \text { and } \\
& \frac{q}{\delta}=\frac{k_{b 2}}{s^{2} / \omega_{n b}^{2}+2 \xi / \omega_{n b}+1} .
\end{aligned}
$$

Where $f_{z}, q$, and $\alpha$ are the normal acceleration, body rate, and angle of incidence, $k_{b 1}$ and $k_{b 2}$ are the airframe aerodynamic steady state gains, $\omega_{n b}$ is the airframe natural frequency, $\xi$ is its damping factor, and $\mathrm{s}$ is the Laplace operator. The system parameters had been obtained lby the FFT of the transient response and extraction of the poles location is made via MATLAB tool box [9].

In the present paper the work is extended to obtain the complete airframe transfer function. Fig.(6a) and Fig. (6-b) show a comparison between the transient response obtained from the numerical model and that obtained via Equation (6).

It is clear that the behavior of the approximated functions differ from those of the computer simulation in the transient region. The difference is attributed to the neglection of the zeros in the numerator[5]. Thus, two complex conjugate zeros are included in Eq. 6). The location of the two zeros are obtained via a time-domain matching technique. In this technique, a search for the zeros is

made in the complex plane such that $\int_{0}^{t_{s s}}\left|\Psi_{j m}-\Psi_{j p}\right| d t$ is minimized.

Where $\Psi_{\mathrm{jn}}$ and $\Psi_{\mathrm{jp}}$ are the numerical model and predicted transfer function time response results; respectively. Thus, the improved predicted transfer function of the normal acceleration and body rate can be written as:

$$
\begin{aligned}
& \frac{f_{z}}{\delta}=\frac{\left(S+j z_{1}\right)\left(S-j z_{1}\right)}{s^{2} / \omega_{n b}^{2}+2 \xi / \omega{ }_{n b}+1} \\
& \text { and } \\
& \frac{q}{\delta}=\frac{\left(S+j z_{2}\right)\left(S-j z_{2}\right)}{s^{2} / \omega_{n b}^{2}+2 \xi / \omega_{n b}+1} .
\end{aligned}
$$

Fig. (7-a) and Fig. (7-b) show the comparison between results of the numerical simulation and the predicted transfer functions. 


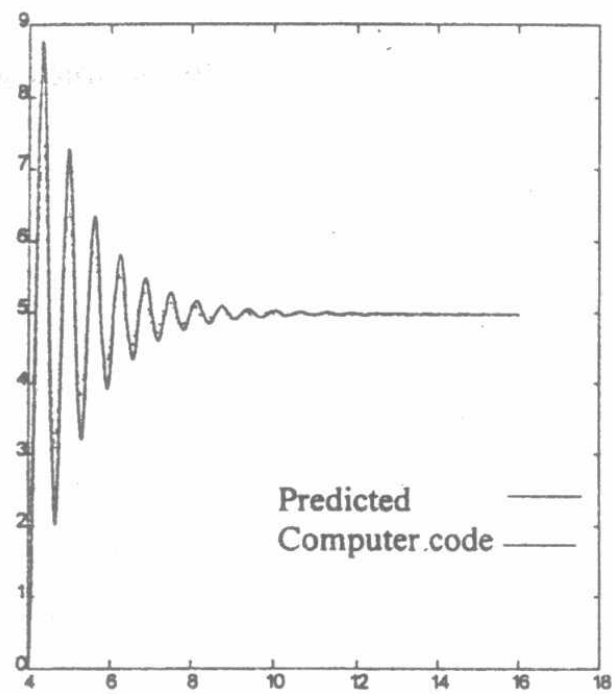

Fig,(6.a) Comparison of response carried out from the computer code and the predicted transfer function given by equation (6.a).

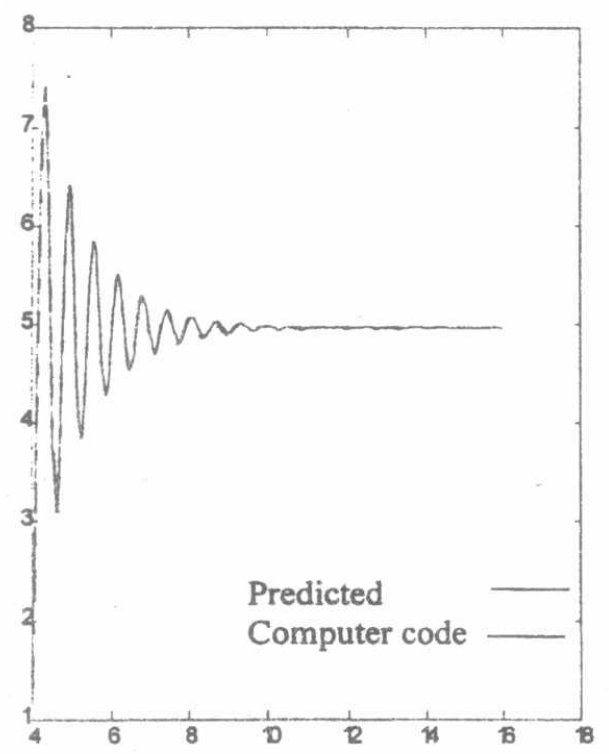

Fig,(7.a) Comparison of response obtained Fig,(7.b) Comparison of response obtained from the computer code and the predicted from the computer code and the predicted transfer function given by equation (7.a).

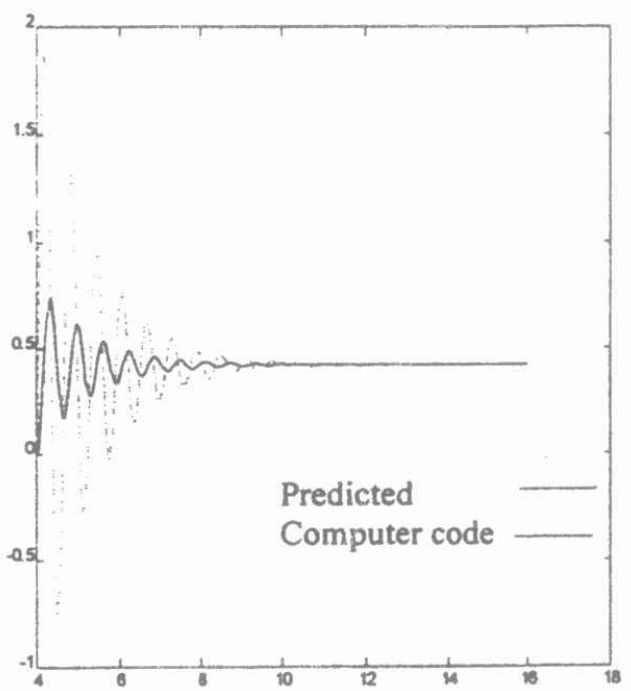

Fig,(6.b) Comparison of response carried out from the computer code and the predicted ${ }^{\prime}$ transfer function given by eqaution (6.b).

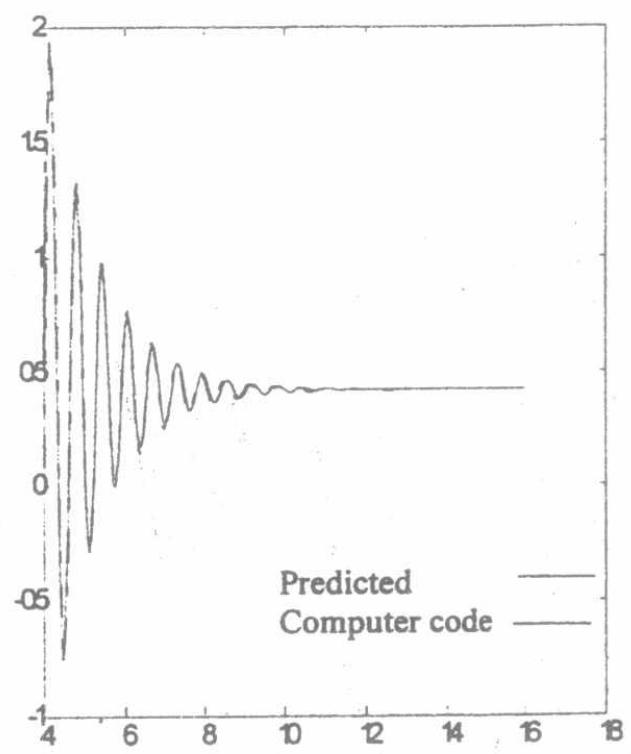

transfer function given by equation (7.b). 
Fig. (8) shows the airframe transfer function pole an zero locations in the complex s-plane as the mach number increases from 2 to $4 . \mathrm{M}$.

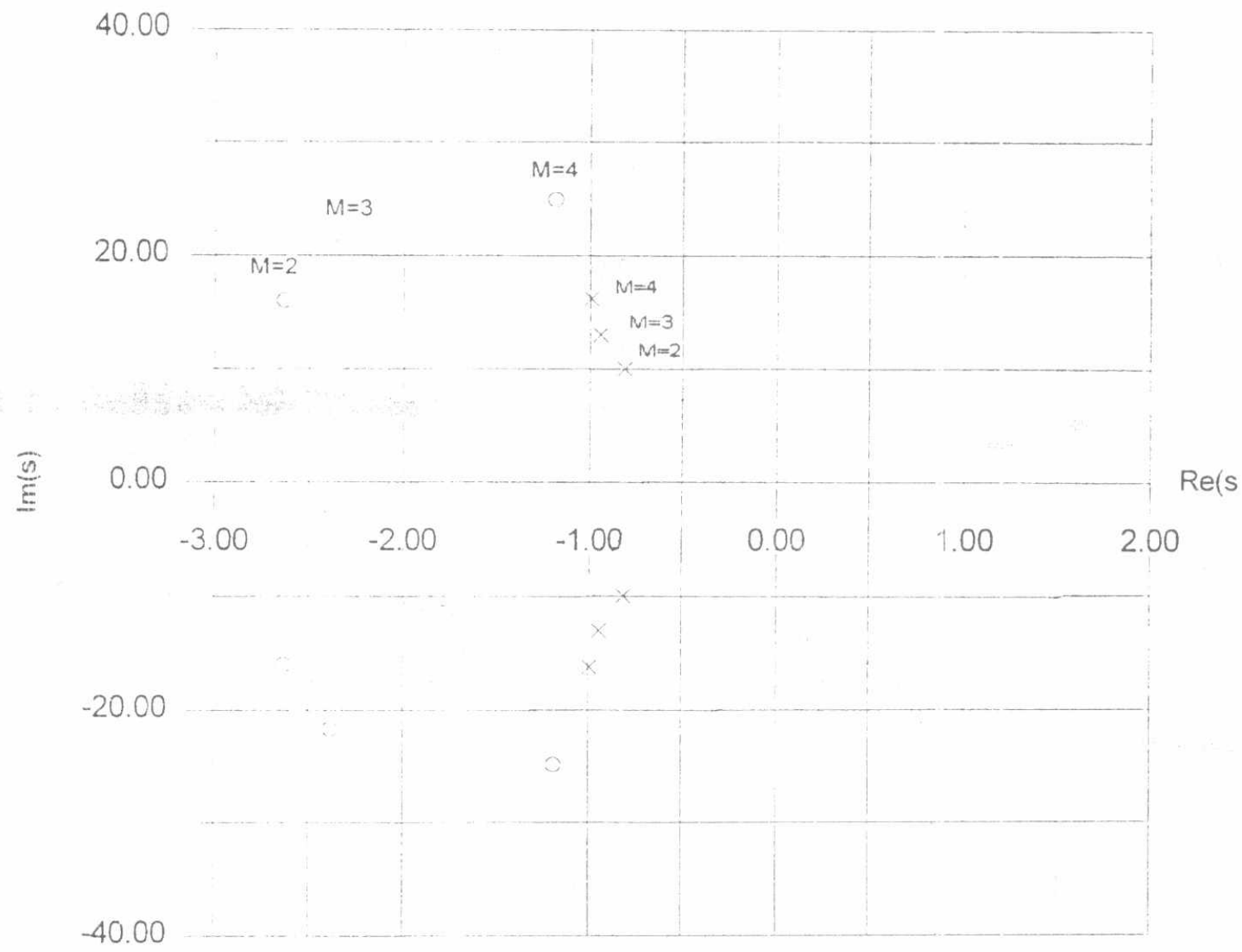

Fig. (8) Pole-Zero locations in the complex s-plane of the airframe response for different Mach numbers

It is apparent that as the mach number increases, the complex poles move away from the imaginary axis and the real axis. The pole trajectories as the mach number increases shows that the damping coefficient $(\zeta)$ decreases and the undamped natural frequency $\left(\omega_{n}\right)$ increases.

The presence of zeros in the transfer function of the airframe reduces the peak overshoot of the airframe oscillations. The reduction level depends on the real part of zeros. Thus, as mach number increases the peak overshoot increases and the airframe becomes less stable. In the mean time, the presence of these zeros increases the airframe response to high frequency' steering commands.

The autopilot will be then considered. Fig. (9) shows the employed autopilot. An accelerometer and a rate gyro are used to improve the airframe response. The two devices are located at the missile C.G. 


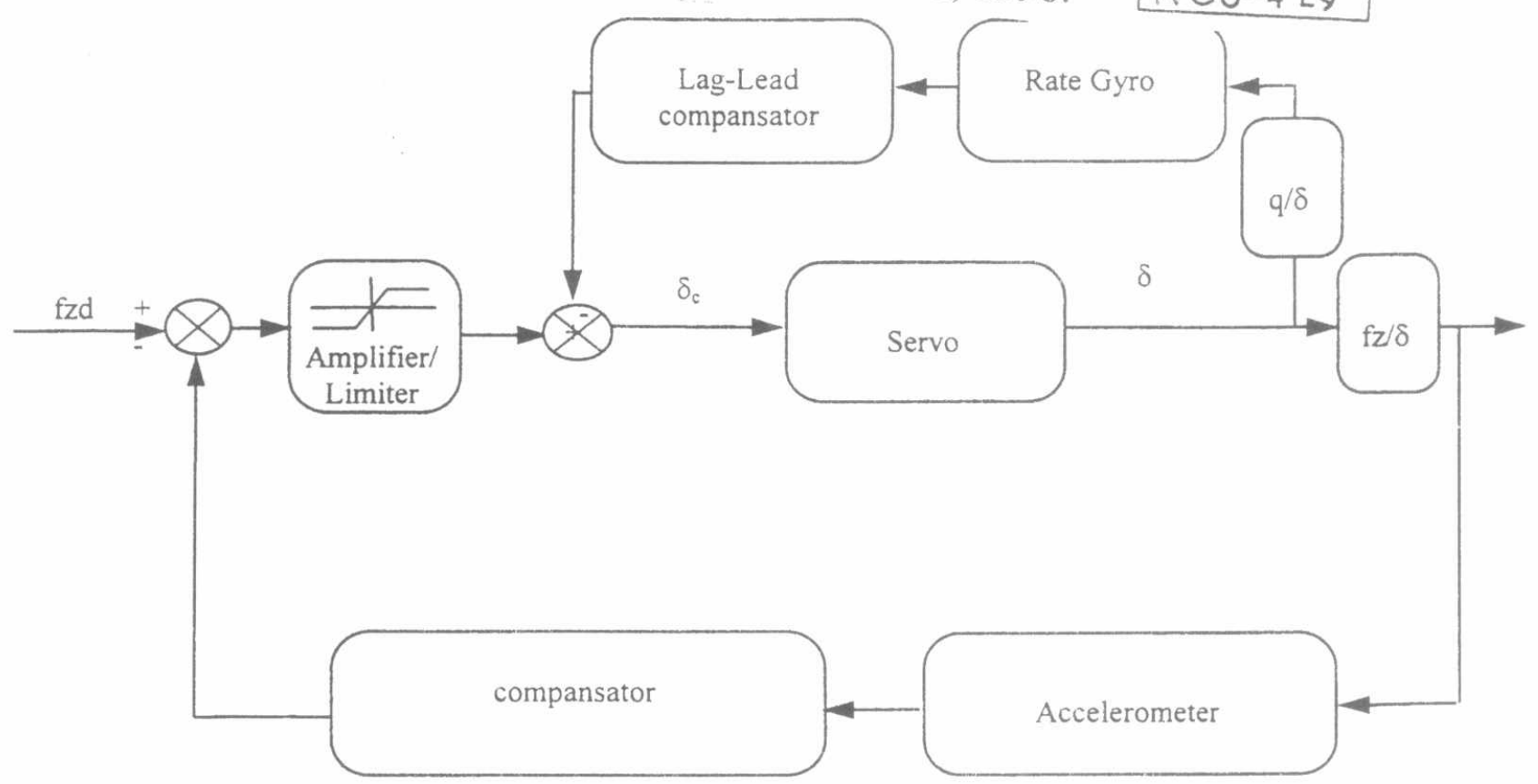

Fig. (10) Block diagram of the autopilot element characteristics.

The transient analysis carried out on the airframe will be applied again on the autopilot. The autopilot of the missile is excited by applying input error signal of 1 volt at the instant $t_{0}$ as :

$$
K_{1}(t)=1 . \mathrm{U}\left(t-t_{o}\right) \text {. [Volt] }
$$

The normal acceleration, the incidence angle, and the body rate in the pitch plane ate displayed versus time for different velocity values given by Mach number $M=2, M=3$, and $M=4$; respectively. Illustrations of the transient responses are shown in Fig. (9). The increase of the Mach number from 2 to 4 results in $56 \%$ increase in the autopilot aerodynamic gain. Referring to Figs. (6) and (7), the same increase in the Mach number results in $81 \%$ increase in the aerodynamic gain of the airframe. This illustrates one of the major advantages of utilizing the autopilot. If this percentage variation in the steady-state gain is not acceptable by the overall system requirements, a dynamic pressure sensor may be employed to sense the variation in the Mach number and then control the gain of one of the blocks in the autopilot loop [10][11].

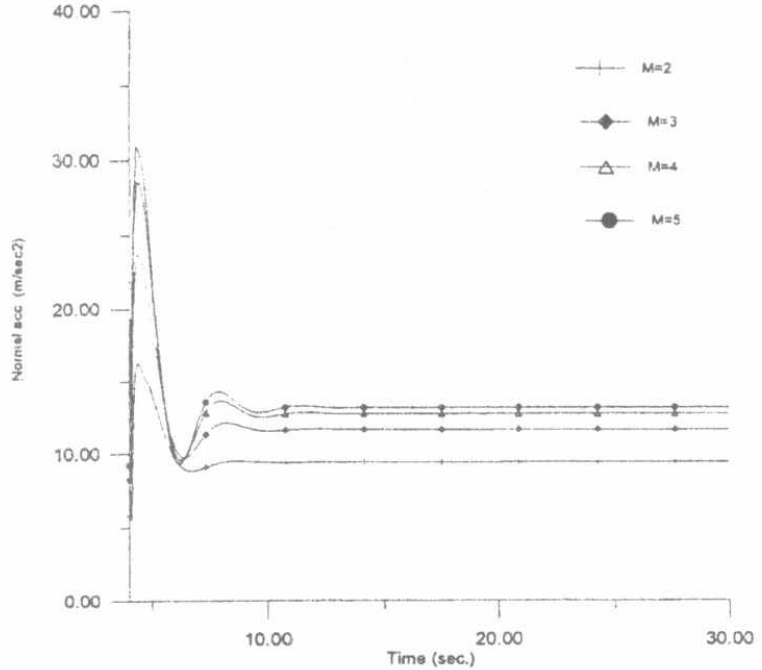

Fig. (9.a) The normal acceleration response due to unit step error signal applied to the autopilot for various mach numbers.

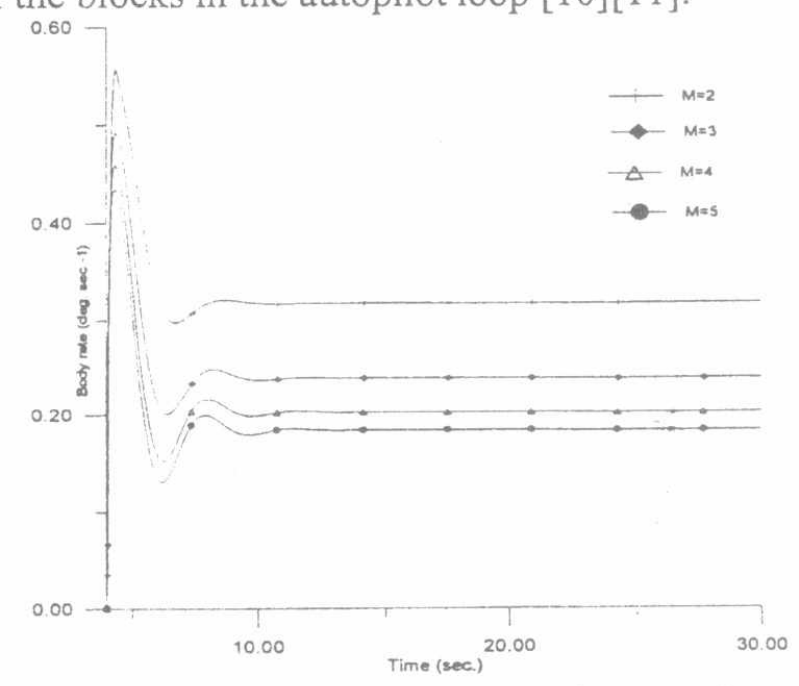

Fig. (9.b) The body rate response due to unit step error signal applied to the autopilot for various mach numbers. 
Proceeding of the $1^{\text {st }}$ ICEENG conference, 24-26 March, 1998.

By using the airframe transfer function (Eq. (7)), and ignoring the effect of the limiter shown in (Fig. 9 ), the pole-zero location of the autopilot response $\left(\frac{f_{z}}{f_{z d}}\right)$ in the complex s-plane can be calculated. Table (1) shows the computed poles and zeros at different mach numbers. It is clear that small variation in the location of these poles and zeros is caused as the Mach number increases. This is an important advantage of the autopilot since the system dynamical behavior becomes insensitive to environmental conditions variation throughout the missile flight mission. Thus, the design of more robust guidance loop becomes more feasible.

Table. 1. The Pole-Zero location of the autopilot transfer function in the complex s-plane.

\begin{tabular}{|c|c|c|c|}
\hline \multirow{2}{*}{$\begin{array}{c}\text { Pole } \\
\text { No. }\end{array}$} & $\mathbf{M}=\mathbf{2}$ & $\mathbf{M}=\mathbf{3}$ & $\mathbf{M}=\mathbf{4}$ \\
\hline & -100.003 & -100.05 & -100.06 \\
\hline 6 & -19.01 & -18.9 & -18.83 \\
\hline 6 & $-3.86 \pm \mathrm{j} 18.27$ & $-2.41 \pm \mathrm{j} 17.74$ & $-0.59 \pm \mathrm{j} 18.75$ \\
\hline 4 & $-1.68 \pm \mathrm{j} 10.39$ & $-3.32 \pm \mathrm{j} 13.97$ & $-5.14 \pm \mathrm{j} 15.03$ \\
\hline 3 & $-0.81 \pm 9.97$ & $-0.93 \pm \mathrm{j} 13.00$ & $-0.91 \pm \mathrm{j} 15.05$ \\
\hline 2 & $-0.91 \pm 1.69$ & $-.92 \pm \mathrm{j} 1.68$ & $-.73 \pm \mathrm{j} 1.92$ \\
\hline 1 & -1.81 & -1.81 & -2.15 \\
\hline Zero & & Zeros & \\
\hline No. & & -19.2308 & -19.2308 \\
\hline 6 & -19.2308 & $-4.6296 \pm \mathrm{j} 18.6799$ & $-4.6296 \pm \mathrm{j} 18.6799$ \\
\hline 5 & $-4.6296 \pm \mathrm{j} 18.6799$ & $-2.6316 \pm \mathrm{j} 16.0073$ & $-1.087 \pm \mathrm{j} 24.8985$ \\
\hline 4 & $-2.6316 \pm \mathrm{j} 16.0073$ & $-0.9432 \pm \mathrm{j} 12.9847$ & $-0.9136 \pm \mathrm{j} 15.0479$ \\
\hline 3 & $-0.81 \pm \mathrm{j} 9.9671$ & $-1.4 \pm 1.4283$ & $-1.4 \pm 1.4283$ \\
\hline 2 & $-1.4 \pm 1.4283$ & -0.8475 & -0.8475 \\
\hline 1 & -0.8475 & & \\
\hline
\end{tabular}

Fig. (11) shows the pole-zero locations of the autopilot in the complex s-plane at mach number equals 2. It is noted that each pole has a close neighbor zero. Thus, the damping contribution of this poles at high frequency is reduced. The pole that is far from the $\operatorname{Im}(s)$ axis $(p 7)$ limits the effect of the high frequency noise on the system performance. Inspection of Fig. (11), reveals that the dominant poles are P1, P2, P3, P4 and P5 since they are very close to the $\operatorname{Im}(\mathrm{s})$ axis. The presence of $\mathrm{Z} 1, \mathrm{Z2}, \mathrm{Z} 3$ and $\mathrm{Z} 5$ very close to the poles, neutralize their effect. Thus, the only effective poles and zeros are those given by P4, P4* $, \mathrm{Z4}, \mathrm{Z4} 4^{*}$ and P7. The overall transfer function of the autopilot can then be written as :

$$
\frac{f_{y}}{f_{y d}}=\frac{(s-Z 4)\left(s-Z 4^{*}\right)}{(s-P 4)\left(s-P 4^{*}\right)(s-P 7)} .
$$


Proceeding of the $1^{\text {st }}$ ICEENG conference, 24-26 March, 1998. RG6-439

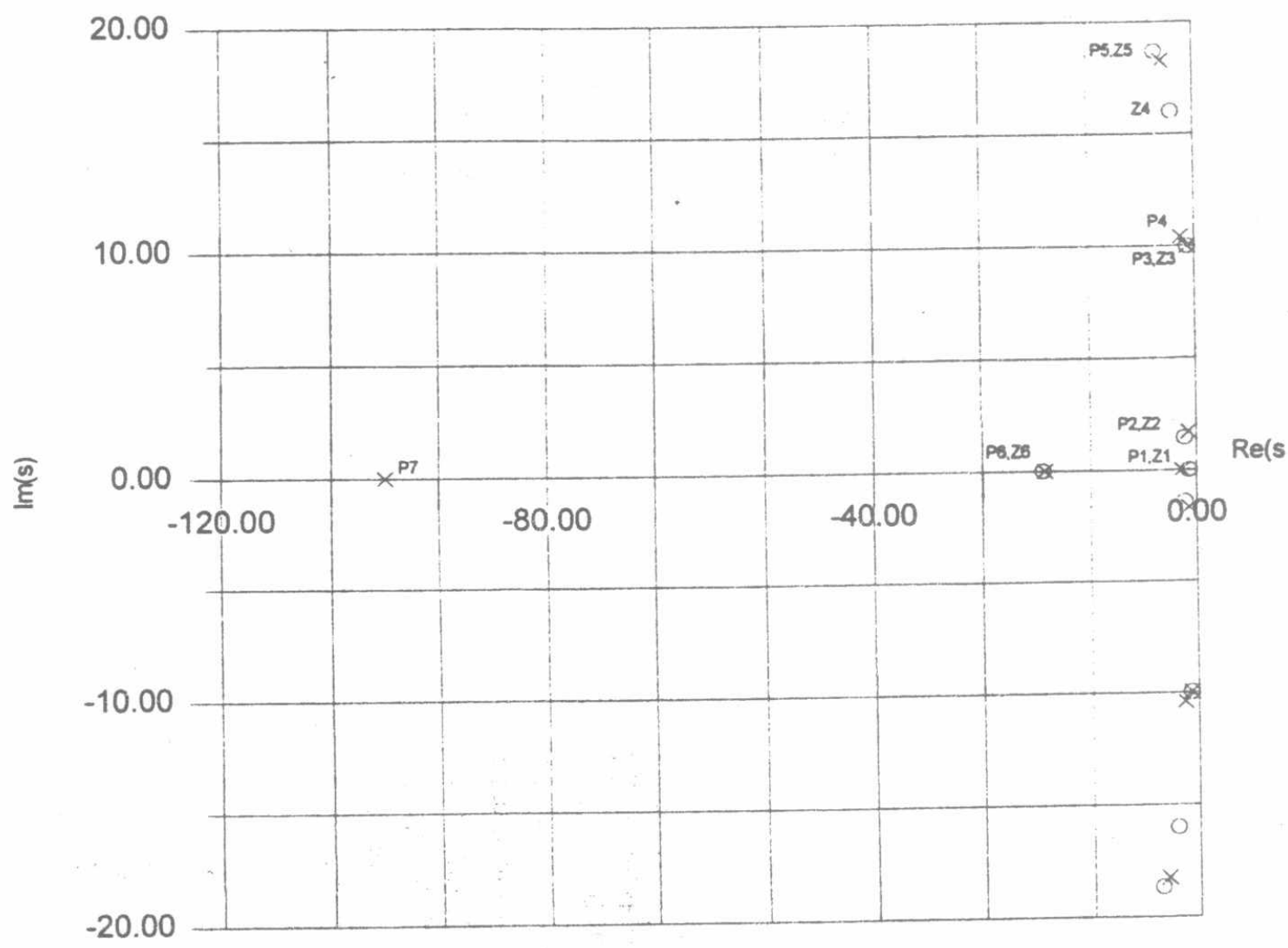

Fig. 11. The Pole-Zero location of the autopilot response in the complex s-plane at mach number

$$
\mathrm{M}=2
$$

The frequency response of the autopilot, obtained from the FFT of the time domain transient response is shown in Figs. (12-a) and (12-b).
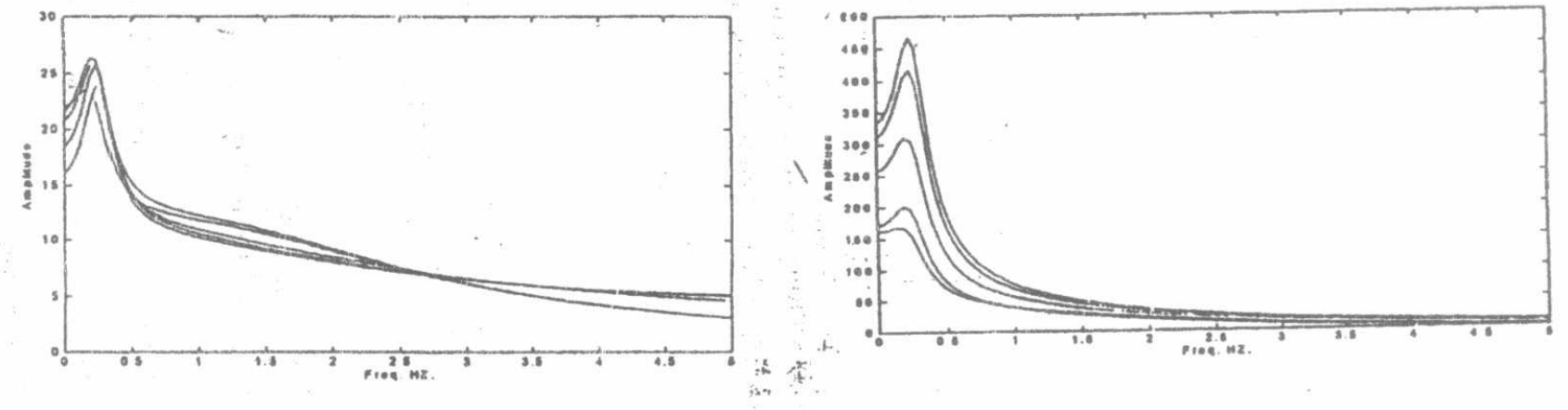

Fig. (12-a) The amplitude spectrum of the missile body rate in the autopilot for different Mach numbers.
Fig. (12-b) The amplitude spectrum of the missile normal acceleration in the autopilot for different Mach numbers.

The presence of the zeros $\mathrm{Z4}$ and $\mathrm{Z4} 4^{*}$ widens the bandwidth while the presence of the pole P7 limits the response at very high frequencies. A comparison between the airframe spectrum and the autopilot spectrum is shown in Fig. (13) which highlights the broadening of the autopilot spectrum. 
Proceeding of the $1^{\text {st }}$ ICEENG conference, 24-26 March, 1998. [R66-632

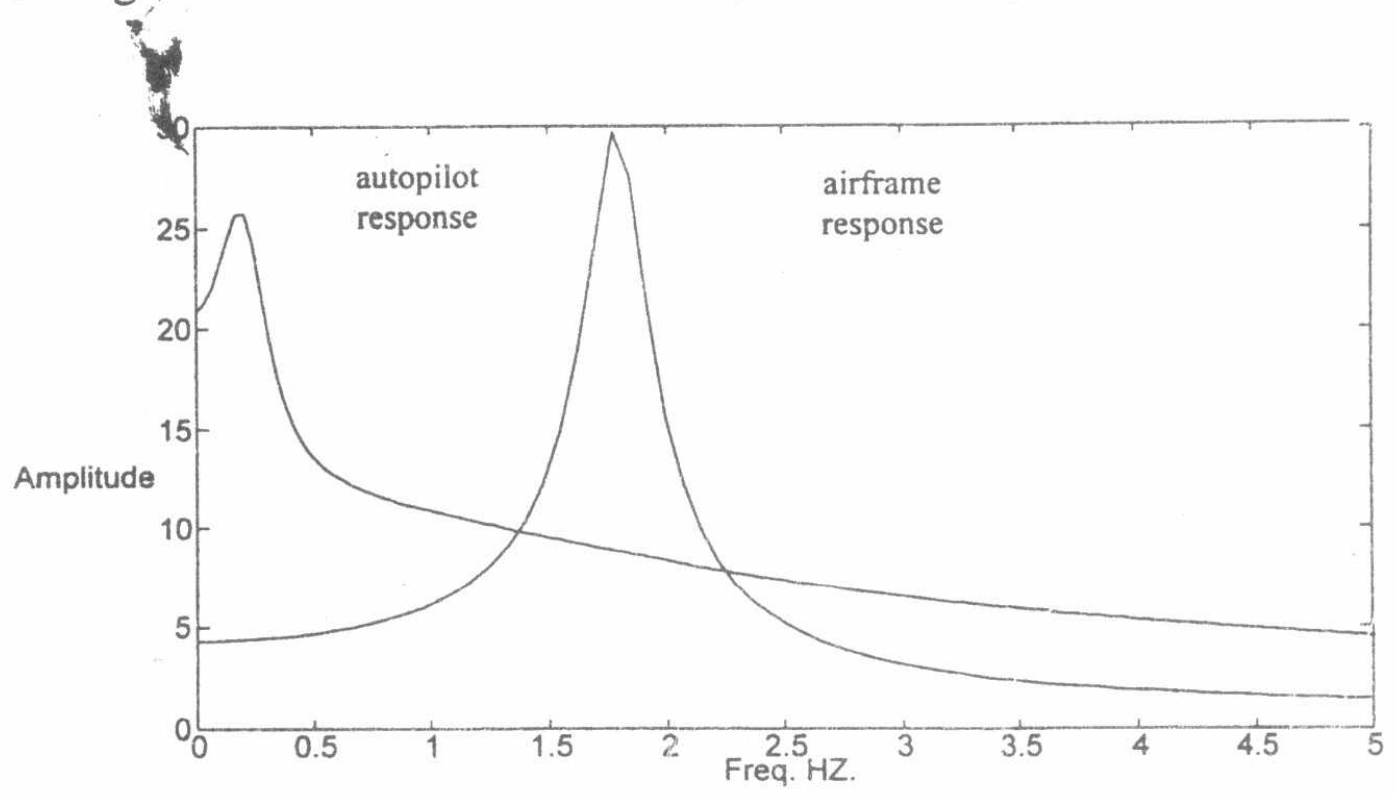

Fig. 13. Comparison of the amplitude spectrum of the airframe and the: autopilot.

\section{CONCLUSION AND FUTURE WORK}

The missile airframe analysis initiated in [1] is continued. An accurate prediction to the airframe transfer characteristics is carried out. The analysis is extended to cover the missile autopilot. The adavantages of having autopilot on the missile board are pointed out. The analysis of the complete guidance loop represents natural extension to the present work in the future.

\section{REFERENCES}

[1] Y.Z. EL-Halwagy; M.S. Ali; and M.S. Ghoniemy "Command Guidance System : Airframe Analysis", $7^{\text {th }}$ ASAT Conf., May 1997.

[2] Garnell P. and D, J. East, "Guided Weapon Control Systems", 2nd ed., Pergaman press, New York, 1980.

[3] Philip C. G. "Guidance Simulation Techniquies", AGARD Lecture Series No. 101, May 1979.

[4] Erwin. Kreyszing, "Advanced Engineering Mathematics”, John Wiley \& Sons, Inc. (1994).

[5] Richard C. D. and Robert H. B. "Modern Control Systems", 7th ed., Addison-Wesley, Inc., (1995).

[6] Y.Z. EL-Halwagy; "Simulation and Modelling of Command Guidance System", M.Sc., 1997.

[7] Emil J. E., Jr., "Test And Evaluation Of The Tactical Missile", Volume 119, American Institute of Aeronautics and Astronautics, Inc., (1989).

[8] Robert H. M. Macfadzean, "Surface-Based Air Defense System Analysis", John Wiley \& Sons, Inc., (1995).

[9] Thomas P. K., Loren S., and John N. L. "Signal Processing Toolbox", The Math Works Inc. (1993).

[10] John H. B. "Automatic Control Of Aircraft And Missiles", 2nd ed., John Wiley \& Sons, Inc. (1991).

[11] Zarcan, Paul, "Tactical and Strategic Missile Guidance", In progress in Aeronautic and Astronautics, Vol. 124. Washington, DC, AIAA. (1990). 